\title{
Plasmodium falciparum Cyclic Amine Resistance Locus (PfCARL), a Resistance Mechanism for Two Distinct Compound Classes
}

\section{Citation}

Magistrado, Pamela A., Victoria C. Corey, Amanda K. Lukens, Greg LaMonte, Erika Sasaki, Stephan Meister, Melanie Wree, Elizabeth Winzeler, and Dyann F. Wirth. 2016. "Plasmodium falciparum Cyclic Amine Resistance Locus (PfCARL), a Resistance Mechanism for Two Distinct Compound Classes." ACS Infectious Diseases 2 (11): 816-826. doi:10.1021/acsinfecdis.6b00025. http://dx.doi.org/10.1021/acsinfecdis.6b00025.

\section{Published Version}

doi:10.1021/acsinfecdis.6b00025

\section{Permanent link}

http://nrs.harvard.edu/urn-3:HUL.InstRepos:29626154

\section{Terms of Use}

This article was downloaded from Harvard University's DASH repository, and is made available under the terms and conditions applicable to Other Posted Material, as set forth at http:// nrs.harvard.edu/urn-3:HUL.InstRepos:dash.current.terms-of-use\#LAA

\section{Share Your Story}

The Harvard community has made this article openly available.

Please share how this access benefits you. Submit a story.

Accessibility 


\title{
Infectious

\section{Plasmodium falciparum Cyclic Amine Resistance Locus (PfCARL), a Resistance Mechanism for Two Distinct Compound Classes}

\author{
Pamela A. Magistrado, ${ }^{\dagger, \|}$ Victoria C. Corey, ${ }^{\ddagger}, \|$ Amanda K. Lukens, ${ }^{\dagger, \S}$ Greg LaMonte, ${ }^{\ddagger}$ Erika Sasaki,
} Stephan Meister, ${ }^{\ddagger}$ Melanie Wree, ${ }^{\ddagger}$ Elizabeth Winzeler, ${ }^{\ddagger}$ and Dyann F. Wirth ${ }^{*},, \S$

\author{
${ }^{\dagger}$ Department of Immunology and Infectious Diseases, Harvard T. H. Chan School of Public Health, Boston, Massachusetts 02115, \\ United States \\ ${ }^{\ddagger}$ School of Medicine, University of California-San Diego, La Jolla, California 92093, United States \\ ${ }^{\S}$ Infectious Disease Program, The Broad Institute, 415 Main Street, Cambridge, Massachusetts 02142, United States
}

\section{Supporting Information}

ABSTRACT: MMV007564 is a novel antimalarial benzimidazolyl piperidine chemotype identified in cellular screens. To identify the genetic determinant of MMV007564 resistance, parasites were cultured in the presence of the compound to generate resistant lines. Whole genome sequencing revealed distinct mutations in the gene named Plasmodium falciparum cyclic amine resistance locus ( $p f c a r l)$, encoding a conserved protein of unknown function. Mutations in pfcarl are strongly associated with resistance to a structurally unrelated class of compounds, the imidazolopiperazines, including KAF156, currently in clinical trials. Our data demonstrate that pfcarl

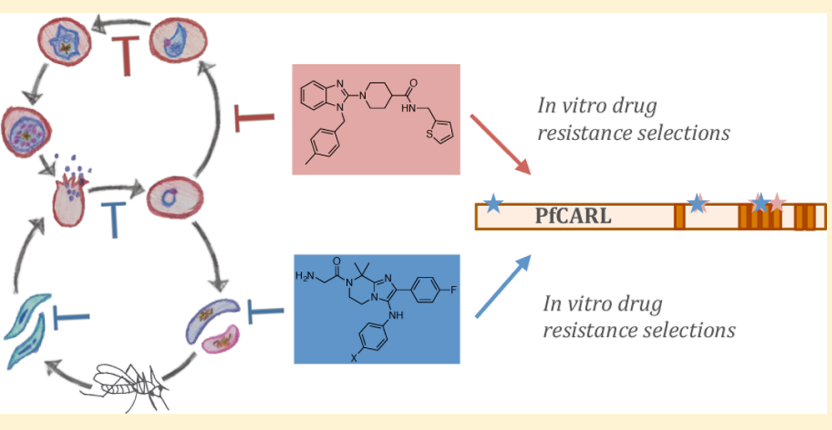
mutations confer resistance to two distinct compound classes, benzimidazolyl piperidines and imidazolopiperazines. However, MMV007564 and the imidazolopiperazines, KAF156 and GNF179, have different timings of action in the asexual blood stage and different potencies against the liver and sexual blood stages. These data suggest that pfcarl is a multidrug-resistance gene rather than a common target for benzimidazolyl piperidines and imidazolopiperazines.

KEYWORDS: malaria, resistance, antimalarials, mutation, PfCARL

$\mathrm{O}$ ver the past 15 years, malaria control measures, including insecticide-treated bed nets, insecticide sprays, and artemisinin-based combinational therapies, have resulted in a reduced global burden of malaria. ${ }^{1}$ Globally, malaria cases have declined by $18 \%$ since the turn of the century, falling from an estimated 262 million in 2000 to 214 million in 2015, and malaria deaths have decreased by $48 \%$, falling from $\sim 839,000$ to $\sim 438,000$ in the same time period. ${ }^{2}$ Of the 100 malaria endemic countries, 35 are pursuing elimination. ${ }^{3}$ Although these results are encouraging, increased parasite resistance to the most effective drug, artemisinin, may undo our progress. ${ }^{4-8}$ Although efforts are being made to restrict the spread of artemisinin-resistant parasites in Southeast Asia, resistance to other standard antimalarials, including chloroquine, pyrimethamine, and sulfadoxine, emerged in the same region of Southeast Asia and subsequently spread to Africa. ${ }^{9}$ Indeed, sporadic reports of artemisinin resistance in Africa and South America may foreshadow a widespread loss of artemisinin efficacy. ${ }^{10,11}$

Anticipating that artemisinin may soon need to be replaced, there has been a concerted effort to place new compounds, especially pan-life cycle compounds with novel mechanisms of action, into the antimalarial drug development pipeline. ${ }^{12}$ One promising new class of multistage malaria drugs is the imidazolopiperazine class, including KAF156, a molecule currently in phase IIb clinical trials for both treatment and prevention of human malaria. KAF156 is well tolerated and orally bioavailable, ${ }^{13}$ and with its potent multistage activity, it has the potential to be the first new antimalarial drug with not only therapeutic but also prophylactic and transmissionblocking activities. KAF156 could thus provide significant aid to malaria eradication. The exact mechanism by which KAF156 exerts its antimalarial activity is unknown, but parasite resistance to KAF156 is associated with the accumulation of mutations in the Plasmodium falciparum cyclic amine resistance locus $(p f c a r l){ }^{14,15} \mathrm{Pfcarl}$ encodes a conserved protein of unknown function with seven conserved transmembrane domains. Analysis of yeast strains in which the pfcarl homologue was deleted ${ }^{16}$ suggests that it plays a role in protein folding within the endoplasmic reticulum. ${ }^{17}$ In addition, the protein has a conserved domain found in other eukaryotic organisms and mutations of the domain result in homeotic

Special Issue: Host-Pathogen Interactions

Received: February 12, 2016

Published: March 28, 2016 


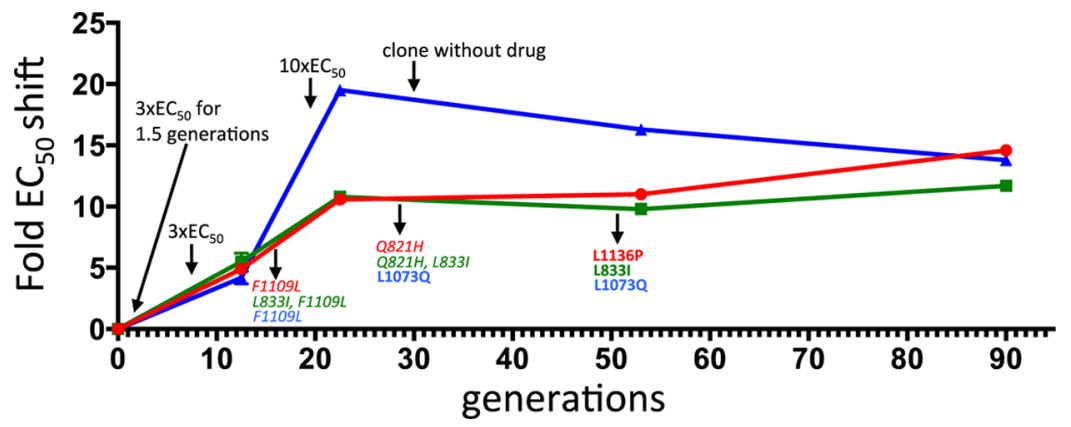

Figure 1. In vitro resistance selection with MMV007564 starting at $3 \times \mathrm{EC}_{50}$ pulse for 1.5 generations followed by continuous drug pressure at $3 \times$ $\mathrm{EC}_{50}$ from generation 7 and $10 \times \mathrm{xEC}_{50}$ from generation 19, resulted in resistant lines as indicated by increasing fold $\mathrm{EC}_{50}$ shift from the $3 \mathrm{D} 7$ parent. Fold $\mathrm{EC}_{50}$ shifts are shown for three independent selections, F1 (red), F2 (green), and F3 (blue). Time points when cloning without drug for singlecell isolation were performed, and pfcarl mutations identified by whole genome sequencing analysis (heterozygous mutations in italics and homozygous mutations in bold) are indicated with arrows.

Table 1. SNV and INDEL Mutation Summary across Generations Passing All Filters

\begin{tabular}{|c|c|c|c|c|c|c|c|c|c|}
\hline & \multicolumn{3}{|c|}{ generation 16} & \multicolumn{3}{|c|}{ generation 28} & \multicolumn{3}{|c|}{ generation 50} \\
\hline & 7564-F1 & 7564-F2 & 7564-F3 & 7564-F1 & 7564-F2 & 7564-F3 & 7564-F1 & 7564-F2 & 7564-F3 \\
\hline genome coverage $(x)$ & 65.15 & 102.48 & 70.1 & 81.34 & 63.85 & 79.76 & 111.06 & 106.81 & 86.12 \\
\hline$\%$ covered by $\geq 15$ reads & 94.6 & 96.7 & 94.9 & 95 & 86.6 & 89.6 & 97.5 & 97.5 & 96.6 \\
\hline \multicolumn{10}{|c|}{ SNVs Identified } \\
\hline total mutations & 6 & 1 & 9 & 0 & 0 & 1 & 2 & 1 & 1 \\
\hline intergenic & 5 & 0 & 5 & 0 & 0 & 0 & 1 & 0 & 0 \\
\hline intronic & 1 & 1 & 4 & 0 & 0 & 0 & 0 & 0 & 0 \\
\hline synonymous & 0 & 0 & 0 & 0 & 0 & 0 & 0 & 0 & 0 \\
\hline non-synonymous & 0 & 0 & 0 & 0 & 0 & 1 & 1 & 1 & 1 \\
\hline genes mutated in all samples & none & & \multicolumn{2}{|c|}{ none } & \multicolumn{3}{|c|}{ Pf3D7_0321900: PfCARL } & & \\
\hline \multicolumn{10}{|c|}{ INDELs Identified } \\
\hline total mutations & 1 & 2 & 2 & 0 & 0 & 0 & 1 & 1 & 2 \\
\hline intergenic & 0 & 0 & 1 & 0 & 0 & 0 & 0 & 1 & 2 \\
\hline intronic & 1 & 2 & 1 & 0 & 0 & 0 & 1 & 0 & 0 \\
\hline synonymous & 0 & 0 & 0 & 0 & 0 & 0 & 0 & 0 & 0 \\
\hline non-synonymous & 0 & 0 & 0 & 0 & 0 & 0 & 0 & 0 & 0 \\
\hline genes mutated in all samples & none & & & none & & & none & & \\
\hline
\end{tabular}

transformations during vertebrate development. ${ }^{18}$ Here we present studies showing that resistance to an alternative compound class with a different phenotypic profile is also conferred through mutations in pfcarl. Specifically, we show that treating parasites with sublethal concentrations of MMV007564, a benzimidazolyl piperidine identified from phenotypic screens (previously reported $\mathrm{EC}_{50}=0.5-0.9 \mu \mathrm{M}$ ) against asexual stages of $P$. falciparum, ${ }^{19,20}$ selects for parasites that have acquired mutations in pfcarl. Investigations into the timing of action in the asexual blood stage and potency in different life cycle stages for MMV007564 and KAF156 reveal that PfCARL is not a common target but a common resistance mechanism for these two chemically distinct compound classes.

\section{RESULTS AND DISCUSSION}

In Vitro Selection Generated Asexual Blood Stage P. falciparum Lines Resistant to MMV007564. The benzimidazolyl piperidine MMV007564 has previously been identified in asexual blood stage screens for activity against the drug-resistant $\mathrm{W} 2\left(\mathrm{EC}_{50}=0.3 \mu \mathrm{M}\right)$ and drug-sensitive 3D7 $\left(\mathrm{EC}_{50}=0.5-0.9 \mu \mathrm{M}\right)$ strains of $P$. falciparum while having low cytotoxicity. ${ }^{19,20}$ However, liver and gametocyte-stage screens show that this compound has limited activity against nonasexual stages. $^{21-26}$ Due to its drug-like properties, asexual blood stage potency in a drug-resistant line, fast/moderate parasite killing rate, ${ }^{27}$ and novel chemical scaffold, we sought to gain insight into benzimidazolyl piperidine mechanisms of action and/or resistance development. We generated MMV007564-resistant (MMV007564 ${ }^{\mathrm{R}}$ ) asexual P. falciparum lines using an in vitro selection method (Figure 1) that has been previously used to associate compounds with their targets. $^{28}$ A clone of the $P$. falciparum reference genome parasite line 3D7 was isolated, and then three independent cultures derived from this clone were grown in the presence of compound at concentrations ranging from 3 to $10 \times \mathrm{EC}_{50}$ $\left(\mathrm{EC}_{50}=513 \pm 56 \mathrm{nM}\right)$. Parasite sensitivity to MMV007564 was evaluated throughout the 2 month selection period. All three MMV007564 ${ }^{\mathrm{R}}$ cultures demonstrated a 4-5-fold shift in $\mathrm{EC}_{50}$ compared to the parental 3D7 at generation 13 (Gen13) after intermittent/continuous compound pressure at $3 \times \mathrm{EC}_{50}$, which increased to a 10-20-fold $\mathrm{EC}_{50}$ shift at Gen23 after continuous compound pressure was increased to $10 \times \mathrm{EC}_{50}$. A single clone for each independent culture was obtained by limiting dilution in the absence of the compound: MMV007564 ${ }^{\mathrm{R}}$-F 1-A3, MMV007564 ${ }^{\mathrm{R}}$-F2-E5, and MMV007564 ${ }^{\mathrm{R}}$-F3-E2. Each clone had at least a 10-fold shift in MMV007564 $\mathrm{EC}_{50}$ compared to the parent (Figure S1). In contrast, there was no shift in $\mathrm{EC}_{50}$ for other antimalarials, including quinine (0.86-1.1-fold shift), atovaquone (1.0-fold shift for all three strains), and mefloquine (0.93-1.0-fold shift), 


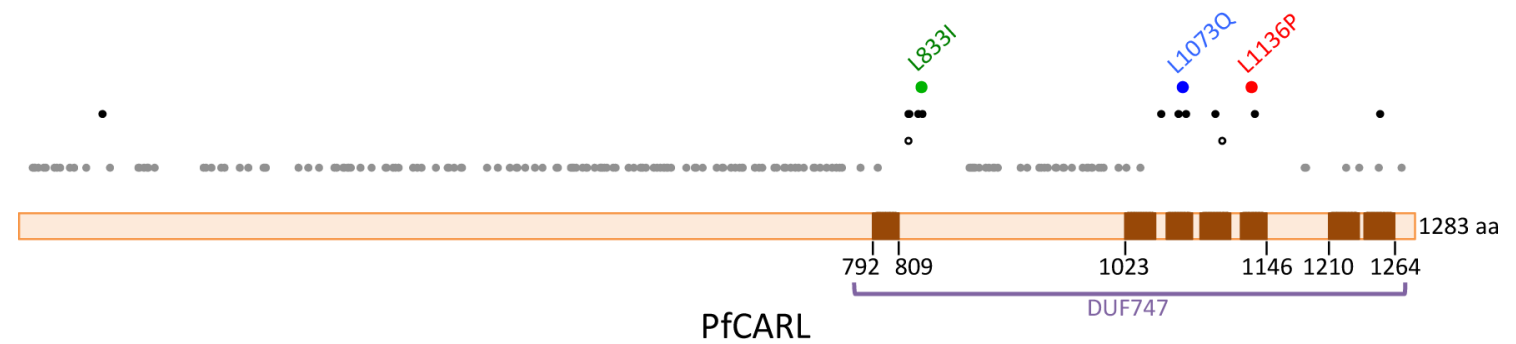

Figure 2. Non-synonymous SNVs in PfCARL: novel SNVs in MMV007564 ${ }^{\mathrm{R}}$ clones F1-A3 (red solid circle), F2-E5 (green solid circle), and F3-E2 (blue solid circle), known SNVs implicated in imidazolopiperazine resistance (black solid circles), ${ }^{14,15}$ SNVs in polyclonal cultures of MMV007564 ${ }^{\mathrm{R}}$ (black open circles) and SNVs in 2517 sequenced global field isolates (gray solid circles). ${ }^{55}$ Transmembrane domains are indicated by brown solid squares, and purple brackets indicate the DUF747 domain. Amino acid mutations are indicated in Table S3.

indicating that resistance was specifically generated to MMV007564 (Figure S1). Additionally, the MMV007564 ${ }^{\mathrm{R}}$ clones had stable resistance to MMV007564 for 37 generations in culture without the compound (Figure 1).

Whole Genome Sequencing (WGS) Identifies pfcarl as the Major Mutated Gene. To determine the genetic determinant of MMV007564 resistance, resistant parasites and the parental clone were analyzed using WGS. First, clonal samples were isolated at Gen30 in the absence of compound pressure and analyzed at Gen50 to identify common genes mutated across all three independent cultures (Figure 1). After the clones had been sequenced to $>60 \times$ coverage using pairedend reads, the sequences were aligned to the $3 \mathrm{D} 7$ reference genome and variants were called. To identify newly emerged genomic changes, the set of variants identified in each resistant clone was compared to the set of variants identified in the parent clone, which was isolated immediately prior to selections. After this comparison, only eight total mutations distinguished the resistant clones from their isogenic parent, and these were evenly split between single nucleotide variants (SNVs) and insertion/deletions (INDELs) (Table 1). INDEL mutations were made up of three intergenic and one intronic mutation, whereas SNVs were composed of one intergenic and three non-synonymous mutations. All three non-synonymous SNVs mapped to a single gene, Pf3D7_0321900, previously named the $P$. falciparum cyclic amine resistance locus ( $p f c a r l$ ). Each clone encoded a different variant amino acid: L833I (7564-F2-E5), L1073Q (7564-F3-E2), and L1136P (7564-F1A3) (Table 1).

To further investigate the temporal acquisition of resistance as well as to determine if the clones we selected at Gen50 were representative of the primary resistance mechanism of MMV007564, we sequenced two additional samples from uncloned cultures taken before the sharp increase in $\mathrm{EC}_{50}$ (Gen16) and immediately before clonal isolation (Gen28). These bulk population samples were sequenced instead of individual clones, as we sought to not only identify when mutations arose but additionally wanted to determine if multiple independent pfcarl mutations could be observed in one selected population.

Variants were identified using the same filters applied to the clonal set, removing positions that did not correspond to at least one sample with a mixed read ratio $<0.2$ (reference allele reads/total reads). This resulted in 15 variants in Gen16 (11 SNVs and 4 INDELs) and one SNV in Gen28 (Table 1; Table S1). Gen 16 was composed of only intronic and intergenic mutations; however, Gen28 contained one non-synonymous pfcarl mutation in flask 3 (L1073Q), the same mutation found at Gen50 in the flask 3 clone (7564-F3-E2). These results indicate that a majority of the pfcarl mutations did not outcompete the entire population, but existed as subpopulations in each flask.

To further examine $p f c a r l$ mutations present in only a subset of the polyclonal population, we reanalyzed mutation calls in both generations while removing the read ratio filter applied previously (Table S2). This resulted in totals of 243 and 84 variants called in Gen 16 and Gen28, respectively. A majority of variants were intergenic or intronic in Gen16, with only 31/243 mutations occurring within an exome region. Conversely, Gen28 had an even distribution of variants, with 49/84 coding region mutations. Three total mixed read SNV calls in pfcarl were identified in Gen16 and Gen28 cultures, with two different SNVs found at each time point. Calculating the binomial distribution, the frequency of mixed-read pfcarl variants was significant in both generations (Gen16, $p=$ 0.000021 ; Gen28, $p=0.000053$ ), suggesting the gene was selected for even in the polygenic populations. Flask 2 contained the SNV L833I at both Gen16 and Gen28 at mixed read ratios of 0.37 (46/124 and 24/64, respectively), which was the primary mutation found in flask 2 at Gen50. All three flasks contained the same nucleotide mutation, resulting in the SNV F1109L at Gen16 at mixed read ratios of $0.68(15 /$ 22), $0.73(90 / 122)$, and $0.25(12 / 48)$ for flasks 1,2 , and 3 , respectively. This mutation was not seen, however, in any of the subsequent generations. Similarly, the SNV Q821H was seen at Gen28 in flasks 1 and 2 at heterozygous read ratios of 0.31 (28/ 88 ) and $0.62(44 / 71)$, respectively, but this mutation was also not seen in any of the other generations (Figure 1).

Our results demonstrate that parasites with the L833Iencoding variant emerged early in the selection experiment but that parasites bearing this mutation did not out-compete other parasites in flask 2. The variant was only identified as a homogeneous call once the culture was cloned. The L1073Qcoding variant likely rose to fixation between generations 16 and 28 in flask 3 , because this mutation was undetected at Gen16, yet had mixed read ratios of 0.00 in Gen28 (0/32) and Gen50 (0/48). In the analysis of the $\mathrm{EC}_{50}$ fold shifts across these generations, it is most likely this mutation occurred between generations 16 and 22, as flask 3 had an observable spike in $\mathrm{EC}_{50}$ during this time. L1136P was found only at Gen50 in flask 1, implying the mutation arose between Gen28 and Gen50. The final two mutations, Q821H and F1109L, were not found at Gen50 but may represent mutations that resulted in initial resistance. F1109L was found in all three flasks but only in Gen16, implying the mutation was out-competed between Gen16 and Gen28. Q821H was found in Gen28, but was not observed in the Gen50 clones that were sequenced. These results demonstrate the importance of analyzing mixed 
Table 2. Cross-Resistance between MMV007564- and Imidazolopiperazine-Resistant Lines with Imidazolopiperazines and Active Benzimidazolyl Piperidines with $\mathrm{EC}_{50}$ Values $<3.5 \mu \mathrm{M}$ against the 3D7 Parent ${ }^{a}$

\begin{tabular}{|c|c|c|c|c|c|c|}
\hline \multirow[b]{2}{*}{ Compound } & \multicolumn{6}{|c|}{$E C_{50}(n M) \pm S D$} \\
\hline & Parent Dd2 & $\begin{array}{c}\text { KAF156 } \\
\text { PfCARL: M81I, } \\
\text { L830V, S1076I }\end{array}$ & Parent 3D7 & $\begin{array}{c}\text { MMVO07564 } \\
\text { PfCARL: L1136P }\end{array}$ & $\begin{array}{l}\text { MMV007564 } \\
\text { PfCARL: } L 8331\end{array}$ & $\begin{array}{l}\text { MMV007564 } \\
\text { PfCARL: } L 1073 Q\end{array}$ \\
\hline \multicolumn{7}{|c|}{ Imidazolopiperazines } \\
\hline KAF156 & $9 \pm 2$ & $3330 \pm 119^{* * * *}$ & $10 \pm 3$ & $6.0 \pm 0.3$ & $48 \pm 5.0^{* * * *}$ & $9 \pm 1$ \\
\hline GNF179 & $3 \pm 1$ & $1020 \pm 120^{* * *}$ & $4.5 \pm 0.2$ & $25 \pm 4.0^{* * * *}$ & $2.25 \pm 0.09$ & $5 \pm 1$ \\
\hline
\end{tabular}

MMV007564 and other benzimidazolyl piperidines with the methyl benzyl of MMV007564 replaced with various chemical groups

\begin{tabular}{ccccccc}
\hline MMV007564 & $342 \pm 145$ & $1620 \pm 134^{* * *}$ & $510 \pm 56$ & $6059 \pm 1415^{* * *} 5360 \pm 848^{* * *}$ & $7320 \pm 287^{* * * *}$ \\
4 & $200 \pm 100$ & $1690 \pm 141^{* * *}$ & $820 \pm 66$ & $5896 \pm 1881^{* *} 5930 \pm 1948^{* *}$ & $5449 \pm 1002^{*}$ \\
5 & $230 \pm 73$ & $1210 \pm 170^{* * *}$ & $893 \pm 118$ & $8200 \pm 1060^{*}$ & $9811 \pm 2542^{* *}$ & $11860 \pm 5906^{* *}$ \\
\hline
\end{tabular}

Benzimidazolyl piperidines with the thiophen of MMV007564 replaced with various chemical groups

\begin{tabular}{lllllll}
\hline 6 & $170 \pm 46$ & $700 \pm 77^{* * *}$ & $415 \pm 30$ & $3510 \pm 476^{* * *}$ & $2050 \pm 41.0^{*}$ & $6999 \pm 1342^{* * * *}$ \\
\hline 8 & $679 \pm 158$ & $1400 \pm 85^{* *}$ & $2222 \pm 593$ & $8321 \pm 1313^{* * *} 8746 \pm 1172^{* * *}$ & $8534 \pm 1977^{* * *}$ \\
9 & $956 \pm 278$ & $2180 \pm 299^{* *}$ & $3428 \pm 960$ & $11120 \pm 5015^{*}$ & $11120 \pm 3662^{*}$ & $9512 \pm 3672^{*}$
\end{tabular}

${ }^{a}$ Statistical analysis was performed using two-tailed $t$ test when comparing mean $\mathrm{EC}_{50}$ values in the KAF156 ${ }^{\mathrm{R}}$ line against the parental Dd2 and ordinary one-way ANOVA followed by Dunnett's multiple-comparisons test when comparing mean $\mathrm{EC}_{50}$ values in three $\mathrm{MMV} 007564^{\mathrm{R}}$ lines against parental $3 \mathrm{D} 7$ ( $*, p<0.05$; **, $p<0.01$; ***, $p<0.001$; ****, $p<0.0001$ ). Light to dark blue shading indicates significant $2-370$-fold $\mathrm{EC}_{50}$ shift compared to parental line.

cultures as well as multiple clones from each population, as it provides a greater understanding of how the mutations developed over time, and some clonal mutations may not have represented the entire population.

Differential Cross-Resistance of MMV007564 and KAF156-Resistant Asexual Blood Stage P. falciparum Lines to Imidazolopiperazines and Benzimidazolyl Piperidines. Previous in vitro selection studies in P. falciparum using the imidazolopiperazines GNF179, ${ }^{15}$ GNF707, GNF452, and $\mathrm{KAF} 156^{14}$ resulted in resistant lines with 13 different mutations in PfCARL (Figure 2; Table S3), occurring either singly or in combination. Except for the Q821H mutation found in the polyclonal MMV007564 ${ }^{\mathrm{R}}$ flask 1 and 2 samples at Gen28, none of the mutations found in the MMV007564 ${ }^{\mathrm{R}}$ cultures overlapped with the PfCARL variants identified in the imidazolopiperazine-resistant lines. ${ }^{14,15}$ The vast majority of the PfCARL resistance mutations for both the imidazolopiperazines and MMV007564 were present at or near the predicted transmembrane domains of the protein, and all PfCARL mutations found in the MMV007564 ${ }^{\mathrm{R}}$ clones (L833I, L1073Q and L1136P) were within 10 bases of suspected resistanceconferring PfCARL alleles (L830 V, E834D, M1069I, S1076N/ $\mathrm{R} / \mathrm{I}$, and $\mathrm{I} 1139 \mathrm{~K})$ that emerged after imidazolopiperazine exposure (Figure 2; Table S3). These data suggest a shared structure-function relationship. Similar to the imidazolopiperazine resistance mutations, ${ }^{14,15}$ PfCARL mutations found in MMV007564 ${ }^{\mathrm{R}}$ lines were in a conserved region of this protein family (DUF747) found across all eukaryotic phyla, suggesting that these resistance loci play an important biological role in PfCARL function.

To test the theory of shared genetic resistance between the two compound classes, we characterized the extent of crossresistance between MMV007564 ${ }^{\mathrm{R}}$ and a triple-mutant KAF156- resistant line $\left(\mathrm{KAF} 156^{\mathrm{R}}\right.$ : M81I, L830V, S1076I) using MMV007564, KAF156, and GNF179 (Table 2). KAF156 and its close analogue GNF179 differ only in the halogen atom attached at the para position in the benzene ring (Figure 3a). ${ }^{14,15}$ Unlike the MMV007564 ${ }^{\mathrm{R}}$ lines, the $\mathrm{KAF} 156^{\mathrm{R}}$ line exhibited cross-resistance to all three compounds. Two of the MMV007564 ${ }^{\mathrm{R}}$ PfCARL mutant lines conferred resistance to either KAF156 (L833I) or GNF179 (L1136P), whereas the MMV007564 ${ }^{\mathrm{R}}$ PfCARL L1073Q mutant was not cross-resistant to either of the imidazolopiperazines tested. This suggests that various PfCARL mutations in the MMV007564 ${ }^{\mathrm{R}}$ lines are compound class specific and have different efficacies in conferring resistance to the imidazolopiperazines tested.

To reveal structure-activity relationships (SAR), commercially available MMV007564 analogues were tested against the parental 3D7 cell line (Figure 3b,c; Table 2; Table S4). The analogues either had the methyl benzyl (substructure A) or the thiophene (substructure B) of MMV007564 replaced by different chemical groups (Figure $3 \mathrm{~b}, \mathrm{c}$ ). A methyl benzyl in substructure A with the methyl at either the meta or para position (MMV007564, 4 and 5) appeared to be important for activity in 3D7 parasites (Table 2; Table S4). In general, having an aromatic ring in substructure B was important for activity (MMV007564, 6-9), but the heteroatom nitrogen (7) or oxygen (9) in the aromatic ring was not preferred (Table 2; Table S4). Next, we tested cross-resistance of PfCARL mutant lines against these analogues. Interestingly, all MMV007564 analogues considered active against parental $3 \mathrm{D} 7\left(\mathrm{EC}_{50}\right.$ values $<3.5 \mu \mathrm{M}$ ) had significantly reduced activity against both the KAF $156^{\mathrm{R}}$ line and all three MMV007564 ${ }^{\mathrm{R}}$ mutants, although at various levels (Table 2). The KAF156 ${ }^{\mathrm{R}}$ line was $\sim 370$-fold resistant to imidazolopiperazines, whereas the $\mathrm{KAF} 156^{\mathrm{R}}$ and MMV007564 ${ }^{\mathrm{R}}$ lines were 2-17-fold resistant to benzimidazolyl 


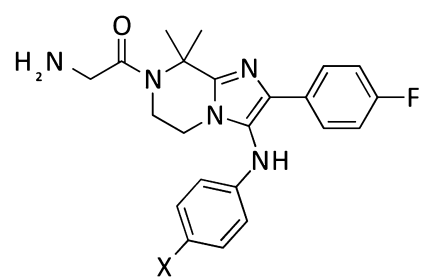

KAF156: $X=F$ GNF179: $X=C l$
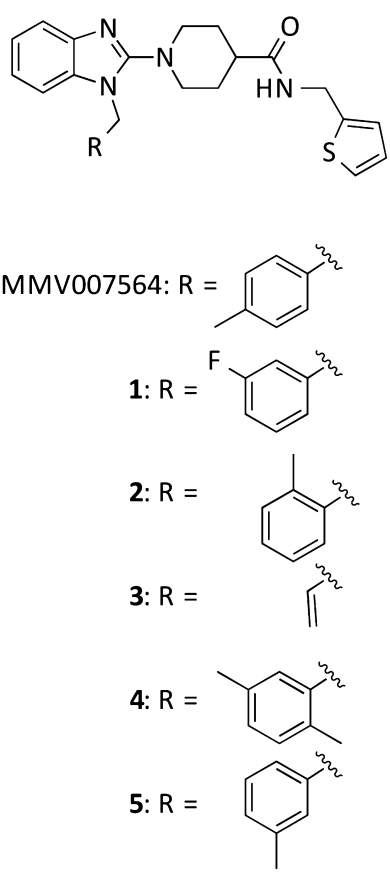<smiles>[R]NC(=O)C1CCN(c2nc3ccccc3n2Cc2ccc(C)cc2)CC1</smiles><smiles>[R]#[R]CCc1ccc(F)cc1</smiles><smiles>[R]#[R]Cc1cccnc1</smiles><smiles>[R]=CCc1ccccc1</smiles><smiles>[R]=C=CCc1ccco1</smiles><smiles>CC(C)CCC=C=[Ru]</smiles><smiles>[Y11]=C=CCC1CCCO1</smiles><smiles>[R]C=CC1CCCCC1</smiles><smiles>CCN(CC)CC[13C]=C[13CH3]</smiles>

14: $\mathrm{R}=\{\mathrm{N} \mathrm{O}$

Figure 3. Chemical structures of imidazolopiperazines and benzimidazolyl piperidines used in this study: (a) imidazolopiperazines; (b) MMV007564 and other benzimidazolyl piperidines where the methyl benzyl group of MMV007564 is replaced with different chemical groups; (c) benzimidazolyl piperidines where the thiophene of MMV007564 is replaced with different chemical groups.

piperidines (Table 2). We speculate that the high level of imidazolopiperazine resistance of the $\mathrm{KAF} 156^{\mathrm{R}}$ line is due to the presence of triple mutations in PfCARL. As shown in previous studies, imidazolopiperazine resistant lines containing multiple PfCARL mutations were most likely to have $\geq 40$-fold imidazolopiperazine resistance in contrast to lines with single PfCARL mutations. ${ }^{14,15}$ In the absence of MMV007564 ${ }^{\mathrm{R}}$ lines with multiple PfCARL mutations, it is not known whether the same is true for benzimidazolyl piperidine resistance. Structural studies will help elucidate how imidazolopiperazine and benzimidazolyl piperidine interact with PfCARL and determine how the various mutations identified in this locus contribute to resistance. Nevertheless, our current data provide novel avenues to pursue SAR.

MMV007564 and Imidazolopiperazines Have Varying Potencies against the Different $P$. falciparum Life Cycle Stages. Because PfCARL mutations mediate resistance of asexual blood stage $P$. falciparum to both benzimidazolyl piperidines and imidazolopiperazines, we hypothesized that these two compound classes share a common target. $P$. falciparum has a tightly regulated intraerythrocytic developmental cycle (IDC) in which proteins reach peak expression at various stages during the cycle. ${ }^{29}$ Parasites are therefore most susceptible to a small molecule inhibitor during the stage of the IDC in which the target of the molecule is expressed and generally unaffected during those stages when the target is not expressed. We therefore sought to determine whether asexual blood stage parasites were most susceptible to both benzimidazolyl piperidines and imidazolopiperazines at the same life cycle stage, suggestive of a shared target (Figure 4; Figure S2). To test this, synchronized 3D7 parasites were incubated with either MMV007564 or GNF179 at various times throughout one complete asexual IDC $(0-46,0-12,12-$ $24,24-36$, or $36-46 \mathrm{~h}$ ), and parasite ring burden was calculated at the 46th hour.

Neither culture treated with compound from ring stage for $46 \mathrm{~h}$ advanced past the trophozoite stage. In addition, there were no obvious morphological differences between the compound-treated parasites. However, when we looked at progression to the ring stage in the next generation, there was a marked difference in the effect of MMV007564 and GNF179 on the IDC at $0-12,12-24$, and $24-36$ h treatments. At $0-12$ $\mathrm{h}$ treatments, MMV007564-treated parasites doubled the ring parasitemia in the next generation, whereas GNF179-treated parasites remained below the starting ring parasitemia. Interestingly, the $12-24$ and $24-36 \mathrm{~h}$ treatments had the opposite effect. At these time points, GNF179-treated parasites at least doubled in the next generation, whereas MMV007564treated parasites remained below the starting ring parasitemia. Therefore, $0-12 \mathrm{~h}$ asexual blood stage was most susceptible to GNF179, whereas 12-36 h asexual blood stage was most susceptible to MMV007564, indicating that these compounds 
a
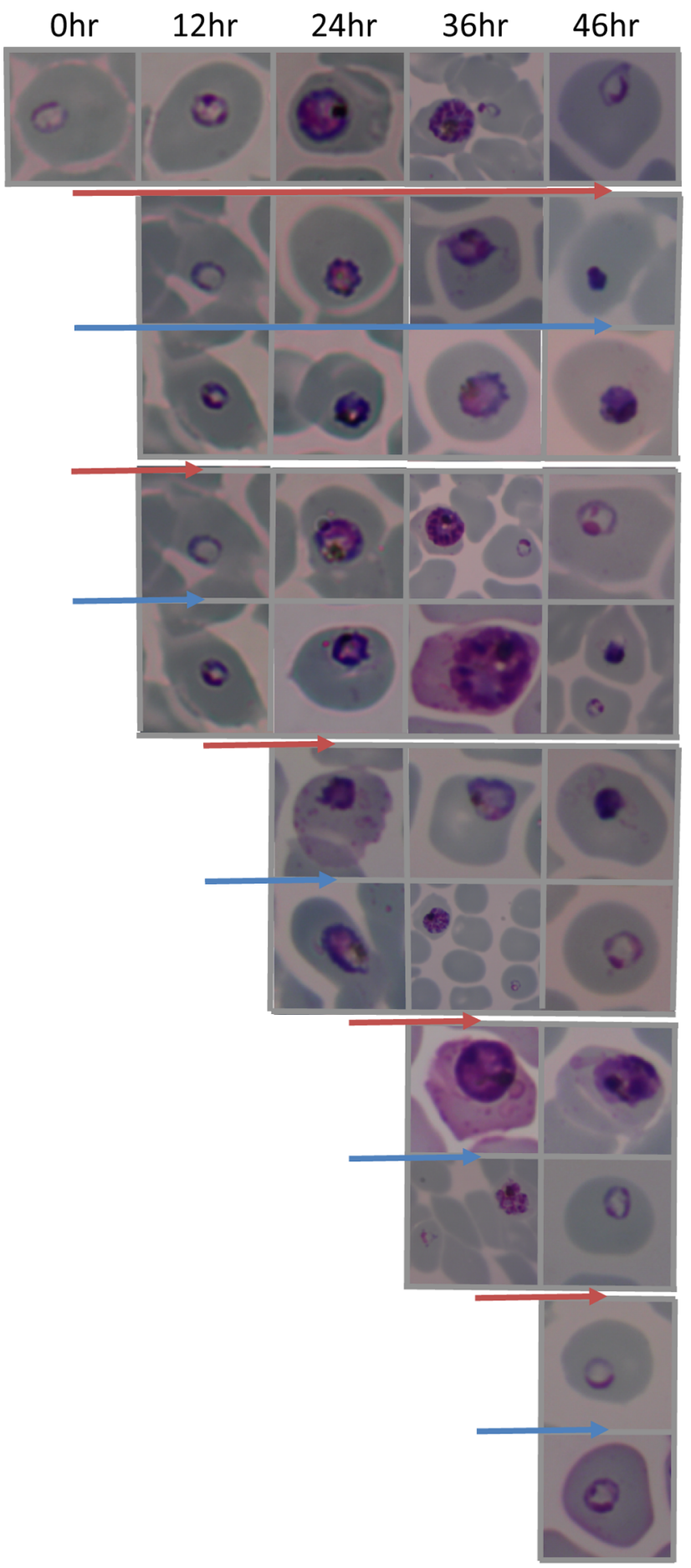

$\mathrm{b}$
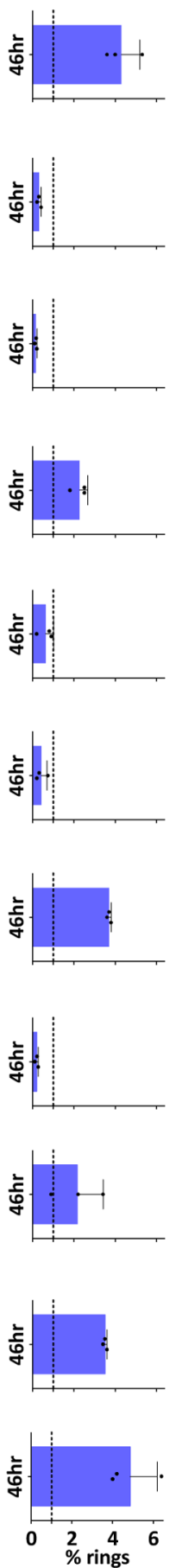

Figure 4. (a) Susceptibility of the different asexual blood stages to $10 \times \mathrm{EC}_{50}$ of MMV007564 (red arrow) and GNF179 (blue arrow) at different time points starting from tightly synchronized, $0-5 \mathrm{~h}$ old rings. Top panel is no compound. Arrows indicate the period when each compound was present in the culture $(0-46,0-12,12-24,24-36,36-46 \mathrm{~h})$. (b) Bars indicate average ring-stage parasitemia at the $46 \mathrm{~h}$ time point, and error bars indicate standard deviation for three independent experiments. Broken lines at $1 \%$ rings indicate starting parasitemia at $0 \mathrm{~h}$.

likely target different proteins essential for asexual blood stage development.

To further define if the compound classes act on two distinct proteins, MMV007564 and KAF156 were assayed against $P$. berghei liver stages and $P$. falciparum stage $\mathrm{V}$ gametocytes ${ }^{30}$ to test for multistage activity (Table 3; Figure S3b,c). KAF156 remained equally efficacious in the liver and sexual stages with
$\mathrm{EC}_{50}$ values not varying significantly from that of the asexual blood stage. In contrast, MMV007564 $\mathrm{EC}_{50}$ values for P. berghei liver stages and P. falciparum stage $\mathrm{V}$ gametocytes are 2- and 5fold higher than the $\mathrm{EC}_{50}$ for the asexual blood stage, respectively. The reduction in MMV007564 efficacy in the sexual blood stage is more pronounced looking at $\mathrm{EC}_{90}$ values, with a 9-fold decrease compared to that of the asexual stage. 
Table 3. Potency of MMV007564 and KAF156 at Different Plasmodium Life Cycle Stages ${ }^{a}$

\begin{tabular}{|c|c|c|c|c|}
\hline \multirow[b]{2}{*}{ prasite life cycle stage } & \multicolumn{2}{|c|}{$\mathrm{EC}_{50}(\mathrm{nM}) \pm \mathrm{SD}$} & \multicolumn{2}{|c|}{$\mathrm{EC}_{90}(\mathrm{nM}) \pm \mathrm{SD}$} \\
\hline & MMV007564 & KAF156 & MMV007564 & KAF156 \\
\hline 3D7 asexual blood stage & $513 \pm 56$ & $10 \pm 3$ & $1010 \pm 58.0$ & $26 \pm 7.0$ \\
\hline P. berghei liver stage & $1250 \pm 248^{* *}$ & $10 \pm 9$ & $2610 \pm 437$ & $13 \pm 11$ \\
\hline P. falciparum stage $\mathrm{V}$ gametocyte & $2706 \pm 400.2 * * * *$ & $4 \pm 1$ & $9121 \pm 4018^{* *}$ & $21 \pm 8.4$ \\
\hline
\end{tabular}

${ }^{a}$ Statistical analyses were performed using ordinary one-way ANOVA followed by Dunnett's multiple-comparisons test to compare mean $\mathrm{EC}_{50}$ values in liver and gametocyte stages against asexual blood stage for each compound (**, $p<0.01$; ****, $p<0.0001)$.

Conclusions. Results of this study suggest that the SNVs observed in pfcarl in our in vitro generated MMV007564 ${ }^{\mathrm{R}}$ lines are the genetic determinants for MMV007564 resistance. We have ruled out pfcarl SNVs as compensatory mutations due to the following observations: (1) mutations in different regions of pfcarl arose independently in three separate selection flasks; (2) MMV007564 ${ }^{\mathrm{R}}$ lines were cross-resistant with imidazolopiperazines, a distinct class of compounds previously shown to have pfcarl mutations as the genetic determinant of resistance; (3) an independently generated imidazolopiperazine-resistant line was cross-resistant with MMV007564 and other benzimidazolyl piperidines; and (4) this and previous studies with various pfcarl mutant lines have found a lack of cross-resistance to standard antimalarials, including mefloquine, artemisinin, ${ }^{14}$ quinine, and atovaquone, implying the cross-resistance seen in this study is specific to the benzimidazolyl piperidine and imidazolopiperazine compound classes.

Experiments to identify the cell cycle window in which each compound exerted its maximal effect revealed that GNF179 and MMV007564 were more effective at different asexual stages, implying that they have different cellular targets. If PfCARL was the direct target of both GNF179 and MMV007564, one would expect the two compound classes to be most effective at similar stages, as previous studies have reported constitutive expression for PfCARL across the IDC with a lack of gene regulation or significant changes in gene expression. ${ }^{29,31,32}$ Therefore, given the varying efficacy observed between the benzimidazolyl piperidine and imidazolopiperazine, PfCARL is most likely a common resistance mechanism. Early asexual ring stages were most susceptible to GNF179, whereas late ring to trophozoite stages were most susceptible to MMV007564. These results may suggest the two compounds target different proteins crucial at different asexual blood stage development or that there is varying permeation of the compounds into the infected RBCs. With MMV007564 having a higher calculated $\log P$ of 4.58 compared to that of KAF156 (2.76) and GNF179 (3.16), it is possible that the early ring stage infected RBCs are permeable enough for GNF179 but not for MMV007564. However, this is inconsistent with our results, where the more permeable late stage infected RBCs are less susceptible to GNF179. Therefore, it is more probable that the various timings of action between MMV007564 and GNF179 are due to having different targets rather than having different permeation properties. Consistent with the possibility that the two compound classes have different targets, MMV007564 had reduced efficacy in the liver and sexual blood stages, whereas KAF156 remained active.

Both the imidazolopiperazine and benzimidazolyl piperidine series possess a six-member ring fused to an imidazole. One of the H-bond acceptor nitrogens occupies the same position in the imidazole for both compound series (Figure 3). However, the pharmacophores of the imidazolopiperazines and the benzimidazolyl piperidines do not overlap. Extensive SAR with imidazolopiperazines has shown that an aniline and an aryl group attached to the imidazole are required for activity, ${ }^{33,34}$ features not present in the benzimidazolyl piperidine series. Given these observations, it may be that PfCARL is a resistance mechanism that prevents the benzimidazolyl piperidines and imidazolopiperazines from acting on their yet unidentified targets.

It is not uncommon for unrelated antimalarials to have common genetic determinants of resistance, reflecting either a multidrug-resistance mechanism or a common drug target. The two most well-studied multidrug-resistance mechanisms involve PfCRT and PfMDR $1,{ }^{35}$ both localized in the digestive vacuole membrane and members of the drug/metabolite and ATPbinding cassette transporter families, respectively. The K76T mutation in PfCRT is a validated marker for chloroquine resistance. PfCRT haplotypes also influence susceptibility to other antimalarials including amodiaquine, quinine, and lumefantrine. Mutations and copy number variations in $p f m d r 1$ have been reported to influence susceptibility to lumefantrine, artemisinin, quinine, mefloquine, halofantrine, and chloroquine. Both PfCRT and PfMDR1 seem to be involved in the transport of these compounds away from their actual targets in the parasite. ${ }^{35,36}$ Recently, drug target identification efforts have identified other common genetic determinants of resistance for unrelated compound classes such as (1) dihydroorotate dehydrogenase (PfDHODH) mutations conferring resistance to alkylthiophenes ${ }^{37-39}$ and triazolopyrimidines; $^{39-42}$ (2) P-type cation transporting ATPase (PfATP4) mutations conferring resistance to spiroindolones, pyrazoles, dihydroisoquinolones, and a number of antimalarial agents in the Medicines for Malaria Venture (MMV) Malaria Box; $^{43}$ (3) cytochrome $b c_{1}$ mutations conferring resistance to atovaquone, ${ }^{44,45}$ decoquinate, ${ }^{46}$ tetracyclic benzothiazepines, ${ }^{47}$ $4(1 \mathrm{H})$ pyridones, ${ }^{48}$ a diversity-oriented synthesis probe, ${ }^{49}$ and quinolone-3-diarylethers; ${ }^{50}$ and (4) phosphatidylinositol-4-OHkinase (PI4K) mutations conferring resistance to imidazopyrazines $^{51}$ and 2 -aminopyradines. ${ }^{52}$ For each of the PfDHODH, PfATP4, cytochrome $b c_{1}$, and PfPI4K inhibitors, there is functional and/or biochemical validation that these unrelated chemotypes directly interact with the mutated target, indicating common targets instead of multidrug-resistance mechanisms. $^{37,38,40,41,43-48,50-52}$ Diverse chemotypes hitting the same targets may indicate that such common targets play critical biological roles and that there are a limited number of accessible drug targets in Plasmodium. Recent in silico target profiling indeed showed that only $4 \%$ of the $P$. falciparum genome is druggable. ${ }^{53}$ This, however, is a conservative estimate because the applied ligand-based prediction is limited to available ligands or orthologous targets in public databases. ${ }^{53}$

The PfCARL mutations we have identified as conferring benzimidazolyl piperidine and imidazolopiperazine resistance have not been observed in 2517 sequenced global field isolates (Figure 2; Table S3). ${ }^{54,55}$ Unlike the resistance mutations, 
which cluster at or near the transmembrane domains, natural diversity is mostly found elsewhere in the protein (Figure 2; Table S3). This suggests that natural polymorphisms in transmembrane regions may be limited due to functional constraints. For the few naturally occurring SNVs at or near the transmembrane domains, it would be beneficial to determine whether the field isolates harboring such mutations are more resistant to benzimidazolyl piperidines and imidazolopiperazines compared with those with mutations in other regions of the protein.

MMV007564 ${ }^{\mathrm{R}}$ and KAF $156^{\mathrm{R}}$ parasites emerged relatively quickly. At $10^{9} 3 \mathrm{D} 7$ inoculum, MMV007564 ${ }^{\mathrm{R}}$ parasites emerged only 15 days after a short pulse at $3 \times \mathrm{EC}_{50}$ followed by $3 \times \mathrm{EC}_{50}$ continuous drug pressure. Similarly, KAF156 parasites emerged after 17 days from a $10^{9} \mathrm{Dd} 2$ inoculum after $3 \times \mathrm{EC}_{50}$ of continuous drug pressure. ${ }^{14}$ This time to resistance evolution in vitro is comparable to that of atovaquone, known to generate resistant parasites readily (16-30 days with $10^{8}$ inoculum $),{ }^{50}$ and relatively fast compared with several other compounds (30-100 days with $10^{9}$ inoculum)..$^{39,47,56,57}$ It should be noted, however, that evolution of resistance depends on parasite strain, concentration of selection agent, and number of mutations required for resistance, making direct comparisons between studies difficult. ${ }^{5,59}$ Furthermore, this and other studies have shown that a single amino acid change in PfCARL is enough to give rise to imidazolopiperazine and benzimidazolyl piperidine resistance in vitro. From these observations, we speculate that imidazolopiperazine and benzimidazolyl piperidine resistance might be easily acquired in the field and/or that there might be pre-existing resistance to these inhibitors. This will have an implication in the field efficacy of KAF156, an imidazolopiperazine currently in clinical trials. Thus, the identification of a suitable partner drug for KAF156 will be crucial to protect its efficacy. This has been effective in prolonging the useful lifespan of atovaquone, which despite resistance readily evolving in vitro, in vivo, and in the field, ${ }^{45,60-62}$ has been used as an effective antimalarial with its partner drug, proguanil. ${ }^{63}$ With regard to the application of MMV007564, the benzimidazolyl piperidine may be useful as a potential asexual antimalarial but, due to its limited activity in nonasexual stages, would not be suitable as a pan-stage candidate.

PfCARL is a conserved protein of unknown function. Previous studies have found that PfCARL homologues may play a role in protein folding within the endoplasmic reticulum in yeast ${ }^{16,17}$ and homeotic transformations during vertebrate development. ${ }^{18}$ Understanding the function of PfCARL will be important to develop strategies that circumvent this common drug-resistance mechanism.

\section{METHODS}

Sources of Compounds and Parasite Lines and Culturing. MMV007564 (synonym GNF-Pf- $4877^{30}$ ) was obtained from GlaxoSmithKline and is freely available as part of the Medicines for Malaria Venture's (MMV) Malaria Box. The following MMV007564 analogues and control antimalarials were commercially obtained: compounds 1 (C7761565), 2 (C776-4170), 3 (D264-0067), 4 (C776-3796; synonym MMV019741, ${ }^{20} \mathbf{5}$ (C776-3971), 6 (C776-3599; synonym GNF-Pf-2535 $\left.{ }^{30}\right), 7$ (C776-3523), 8 (C776-3534), 9 (C776-3561), 10 (C776-3585), 11 (C776-3587), 12 (C7763670), 13 (C776-3575), and 14 (C776-3628) were from ChemDiv, USA; quinine, atovaquone, and mefloquine were from Sigma-Aldrich, USA. The imidazolopiperazines, KAF156 and GNF179, were synthesized as described previously. ${ }^{23,33}$

The following asexual blood stage P. falciparum strains were originally obtained through MR4 as part of the BEI Resources Repository, NIAID, NIH: 3D7, MRA-102, deposited by D. J. Carucci; and Dd2, MRA-156, deposited by T. E. Wellems. The fast growing clone of 3D7, IG06, ${ }^{64}$ was used in this study. The $\mathrm{Dd} 2$ strain resistant to KAF156 and KAF179 was generated in a previous study (clone B3) and sequenced in our laboratory as the strain mutations were previously identified using microarray. ${ }^{15}$ Parasites were cultured by standard methods ${ }^{65}$ in RPMI 1640 medium supplemented with $28 \mathrm{mM} \mathrm{NaHCO}, 25 \mathrm{mM}$ HEPES, $25 \mu \mathrm{g} / \mathrm{mL}$ gentamicin, and $0.5 \%$ AlbuMAX II (Life Technologies 11021-045).

Transgenic hepatoma cells (HepG2-A16-CD81-EGFP) were obtained from the laboratory of Dominique Mazier (INSERM, France). ${ }^{66}$ P. berghei luciferase sporozoites (strain MRA868) were obtained by dissection of infected Anopheles stephensi mosquito salivary glands supplied by the New York University Insectary. P. falciparum stage $\mathrm{V}$ gametocytes were obtained from SANARIA by Dr. Stephen L. Hoffman.

In Vitro Selection of MMV007564-Resistant Asexual Blood Stage $P$. falciparum Lines. To generate MMV007564 ${ }^{\mathrm{R}}$ asexual blood stage P. falciparum lines, an initial intermittent followed by continuous drug pressure was applied to 3D7 (Figure 1). Three culture flasks, equivalent to three independent selections (flasks 1,2, and 3), each with $10^{9} 3 \mathrm{D} 7$ parasites, were pressured with $3 \times \mathrm{EC}_{50}(1.5 \mu \mathrm{M})$ of MMV007564 for 3 days until no live parasites were detected by microscopy. The compound was then washed off, and parasites were allowed to recover, with medium changes every $2-3$ days. When the parasitemia reached $\sim 2 \%\left(10^{9}\right.$ inoculum), the cultures were once again drug-pressured at $3 \times \mathrm{EC}_{50}$. Pressure was subsequently increased to $10 \times \mathrm{EC}_{50}(5 \mu \mathrm{M})$, as the parasites did not die off under continuous drug pressure at $3 \times \mathrm{EC}_{50}$. Due to limitations in MMV007564 availability, the inoculum size was reduced to $5 \times 10^{7}$ at this time. Parasites continued to grow at a typical multiplication rate at $10 \times \mathrm{EC}_{50}$. At different time points during the selection process, in vitro sensitivities to MMV007564 and control antimalarials by a SYBR Green I-based cell proliferation assay were performed to monitor MMV007564 resistance, and aliquots of infected red blood cells (RBCs) were collected for genomic DNA (gDNA) extraction and whole genome sequencing to monitor the acquisition of mutations (Figure 1). After MMV007564 resistance was confirmed for the parasites growing in $10 \times$ $\mathrm{EC}_{50}$, each of the three independent selections was cloned by limiting dilution in the absence of the compound, with an inoculum size of $0.2-0.05$ infected RBCs per well. Parasite clones were detected by microscopy after $\sim 3$ weeks of growth. Resistant parasites were cryopreserved in a solution composed of $28 \%$ glycerol, $3 \%$ sorbitol, and $0.65 \%$ sodium chloride.

Genomic DNA Extraction, Preparation, and Analysis of Sequenced Samples. Parasites were isolated from erythrocytes by washing infected RBCs with $0.05 \%$ saponin. gDNA was subsequently isolated by following standard DNeasy Blood and Tissue Kit protocols (Qiagen). DNA libraries were prepared with the Nextera XT kit (catalog no FC-131-1024, Illumina) using the standard dual index protocol and sequenced on the Illumina HiSeq 2500 using a RapidRun mode and sequencing 100 base pairs deep on either end of the fragments. Paired-end reads were aligned to the $P$. falciparum 3D7 reference genome (PlasmoDB v. 13.0), following the Platypus 
pipeline as previously described. ${ }^{67}$ Mutations, however, including SNVs and insertion/deletions (INDELS), were called using GATK's HaplotypeCaller, filtering mutations based on general recommendations from GATK (Table S2). Samples were additionally filtered by removing positions where read coverage was $<5$ in the parent and any position where all samples had a heterozygous ratio $>0.2$ (reference/total reads).

Binomial Distribution Calculation of Polyclonal Mutations. The binomial distribution was calculated for the mixed read variant calls in both Gen16 and Gen28. Calculations were conducted in Excel with the BIOM.DIST$(x$,trials, $p$, FALSE $)$ function, where $x$ was the number of successes ( 2 for Gen16 and Gen28), trials was the number of total exome region mutations (Gen16 $=31$, Gen28 $=49$ ), and $p$ was the probability of randomly generating a mutation in $p f c a r l$, which was the fraction of bases in pfcarl versus the entire genome (4968/23292622).

Dose Response Assay Phenotyping for $P$. falciparum Asexual Blood Stage. In vitro drug sensitivities of asexual blood stage MMV007564 ${ }^{\mathrm{R}}$ lines, the $\mathrm{KAF} 156^{\mathrm{R}}$ line, and their respective $3 \mathrm{D} 7$ and $\mathrm{Dd} 2$ parents were determined using a SYBR Green I-based cell proliferation assay. ${ }^{68}$ Twelve-point curve dilution series of the test compound were additionally carried out in duplicate or triplicate on the same day and replicated on at least three different days. The $\mathrm{EC}_{50}$ values were calculated using a nonlinear regression curve fit in Prism 5 (GraphPad Software Inc.) Parasite lines were considered resistant if their $\mathrm{EC}_{50}$ values significantly increased at least 2fold from that of the parent.

Timing of Action of MMV007564 and GNF179 in $P$. falciparum Asexual Blood Stage. To compare the timing of action for MMV007564 and GNF179 in P. falciparum asexual blood stages, tightly synchronized $0-5$-h-old ring stage 3D7 parasites were prepared using the method of Witkowski et al. with some modifications. ${ }^{69}$ Schizonts were purified by a 35\%/65\% discontinuous Percoll (Sigma-Aldrich, USA) gradient, washed in RPMI-1640, and cultured for $5 \mathrm{~h}$ to allow reinvasion into fresh RBCs. To remove remaining schizonts and hence purify $0-5$-h-old rings, the culture was treated with $5 \%$ D-sorbitol (Sigma-Aldrich, USA). The purified 0-5-h-old rings were adjusted to $1 \%$ parasitemia and $2 \%$ hematocrit in $1 \mathrm{~mL}$ culture volumes and then incubated for $46 \mathrm{~h}$ until the next ring stage. MMV007564 and GNF179 at $10 \times \mathrm{EC}_{50}(5 \mu \mathrm{M}$ and 40 $\mathrm{nM}$, respectively) were added to the cultures at $0-12,12-24$, 24-36, 36-46, 0-24, 24-46, and 0-46 h. At these drug concentrations, approximately $99.9 \%$ of the parasites are expected to be killed based on a $72 \mathrm{~h}$ drug assay $\left(\right.$ MMV007564 EC E9.9 $=4.4 \pm 1.0 \mu \mathrm{M} ;$ GNF179 $\mathrm{EC}_{99.9}=27$ $\pm 17 \mathrm{nM}$; see Figure S3a for Hill slopes). A no compound control was included. Smears for microscopy were prepared at $0 \mathrm{~h}$ and every $10-12 \mathrm{~h}$ for $46 \mathrm{~h}$. The experiment was replicated on three different days.

Dose Response Assay Phenotyping for Multistage Activity. Liver-stage activity was determined by pretreating hepatic human cells (HepG2) for $2 \mathrm{~h}$ with a 12-point curve dilution series of test compound in 1536-well plates infected with freshly dissected $P$. berghei sporozoites. After $48 \mathrm{~h}$ of incubation, the viability of $P$. berghei exoerythrocytic forms (EEF) was measured by luminescence reaction light output using BrightGlo (Promega). Dose response curves were repeated on three different days to calculate an average $\mathrm{EC}_{50}$ value.
Sexual-stage activity was determined by testing each test compound against late-stage $P$. falciparum gametocytes using a MitoTracker fluorescent-based assay. Briefly, synchronized stage V gametocytes were incubated with compound for 72 h, after which MitoTracker Red CMXRos (Life Technologies) was added to each well together with saponin to lyse the RBCs. Following fixation, plates were imaged using an Operetta High Content Imaging System (PerkinElmer) at fluorescence (590$640 \mathrm{~nm}$ ). High content image analysis software supplied by the Operetta was used to measure the gametocytocidal activity (Harmony, PerkinElmer), with the readout based on the metabolically active gametocyte count per well.

Statistical Analysis. Ordinary one-way ANOVA followed by Dunnett's multiple-comparisons test was used to determine whether there is a significant difference in mean $\mathrm{EC}_{50}$ values between multiple resistant lines and their parent, whereas a two-tailed $t$ test was used for a single resistant line and its parent: ****, $p<0.0001$; ***, $p<0.001$;**, $p<0.01$; $*, p<$ 0.05 .

\section{ASSOCIATED CONTENT}

\section{Supporting Information}

The Supporting Information is available free of charge on the ACS Publications website at DOI: 10.1021/acsinfecdis.6b00025.

Figure S1, comparison of $\mathrm{EC}_{50}$ values for MMV007564, quinine, atovaquone, and mefloquine in MMV007564 selection-generated resistant strains. Figure S2, susceptibility of various asexual blood stages to MMV007564 and GNF179 at $24 \mathrm{~h}$ pulses (0-24 and 24-46 h). Figure S3, dose response curves for MMV007564 and KAF156 for multistage activity. Table S2, summary of applied GATK haplotype filters. Table S4, summary of $\mathrm{EC}_{50}$ values for inactive benzimidazolyl piperidine compounds (PDF)

Table S1, SNV/INDEL results for Gen16, Gen28, and Gen50. Table S3, summary of PfCARL allele mutations identified as conferring benzimidazolyl piperidine and imidazolopiperazine resistance (XLSX)

\section{Accession Codes}

All relevant sequencing samples have been deposited in the National Center for Biotechnology Information (NCBI) Sequence Read Archive database using NCBI accession no. SRP068616.

\section{AUTHOR INFORMATION}

\section{Corresponding Author}

*(D.F.W.) Phone: (617) 432-2214. Fax: (617) 432-4766. Email: dfwirth@hsph.harvard.edu.

\section{Author Contributions}

"P.A.M. and V.C.C contributed equally.

Notes

The authors declare no competing financial interest.

\section{ACKNOWLEDGMENTS}

This work was supported by grants from the Bill and Melinda Gates Foundation (BMGF) (OPP1040406). D.F.W. received support from BMGF Grants OPP1054480, OPP1053604, and OPP1067805 and National Institutes of Health-National Institute of Allergy and Infectious Diseases (NIH-NIAID) Grants R01AI099105 and R01AI093716. P.A.M. and A.K.L. 
received support from the grants of D.F.W. V.C.C received support from a UCSD Genetics Training Program through an institutional training grant from the National Institute of General Medical Sciences (T32 GM008666). E.A.W. received support from two grants from the NIH-NIAID (R01AI103058 and R01AI090141). We thank Tomoyo Sakata-Kato for valuable discussions on SAR and data presentation and Paul Hinkson for excellent technical assistance. We also thank Wes Wong and Angela Early for PfCARL SNV analysis of the Pf3K database. We additionally thank Javier Gamo-Benito and Maria Linares for their support in selecting MMV007564 within the Malaria Drug Target Identification Project consortium.

\section{ABBREVIATIONS}

pfcarl, Plasmodium falciparum cyclic amine resistance locus; $\mathrm{SNV}$, single nucleotide variant; INDELs, insertion/deletions; MMV, Medicines for Malaria Venture

\section{REFERENCES}

(1) Galatas, B., Bassat, Q., and Mayor, A. (2016) Malaria parasites in the asymptomatic: looking for the hay in the haystack. Trends Parasitol. 32, 296.

(2) World Health Organization. (2015) World Malaria Report, Geneva, Switzerland.

(3) Malaria Elimination Initiative. (2015) Country Briefing, MEI, UCSF.

(4) Tun, K. M. (2015) Spread of artemisinin-resistant Plasmodium falciparum in Myanmar: a cross-sectional survey of the K13 molecular marker. Lancet Infect. Dis. 15 (4), 415-421.

(5) Noedl, H., et al. (2008) Evidence of artemisinin-resistant malaria in western Cambodia. N. Engl. J. Med. 359 (24), 2619-2620.

(6) Dondorp, A. M., et al. (2009) Artemisinin resistance in Plasmodium falciparum malaria. N. Engl. J. Med. 361 (5), 455-467.

(7) Ariey, F., et al. (2014) A molecular marker of artemisininresistant Plasmodium falciparum malaria. Nature 505 (7481), 50-55.

(8) Phyo, A. P., et al. (2012) Emergence of artemisinin-resistant malaria on the western border of Thailand: a longitudinal study. Lancet 379 (9830), 1960-1966.

(9) Mita, T., and Tanabe, K. (2012) Evolution of Plasmodium falciparum drug resistance: implications for the development and containment of artemisinin resistance. Jpn. J. Infect. Dis. 65 (6), 465475 .

(10) Borrmann, S., et al. (2011) Declining responsiveness of Plasmodium falciparum infections to artemisinin-based combination treatments on the Kenyan coast. PLoS One 6 (11), e26005.

(11) Vreden, S. G., et al. (2013) Evidence of an increased incidence of day 3 parasitaemia in Suriname: an indicator of the emerging resistance of Plasmodium falciparum to artemether. Mem. Inst. Oswaldo Cruz 108 (8), 968-973.

(12) Flannery, E. L., Chatterjee, A. K., and Winzeler, E. A. (2013) Antimalarial drug discovery - approaches and progress towards new medicines. Nat. Rev. Microbiol. 11 (12), 849-862.

(13) Leong, F. J., et al. (2014) A first-in-human randomized, doubleblind, placebo-controlled, single- and multiple-ascending oral dose study of novel imidazolopiperazine KAF156 to assess its safety, tolerability, and pharmacokinetics in healthy adult volunteers. Antimicrob. Agents Chemother. 58 (11), 6437-6443.

(14) Kuhen, K. L., et al. (2014) KAF156 is an antimalarial clinical candidate with potential for use in prophylaxis, treatment, and prevention of disease transmission. Antimicrob. Agents Chemother. 58 (9), 5060-5067.

(15) Meister, S. (2011) Imaging of Plasmodium liver stages to drive next-generation antimalarial drug discovery. Science 334, 1372.

(16) Winzeler, E. A., et al. (1999) Functional characterization of the S. cerevisiae genome by gene deletion and parallel analysis. Science 285 (5429), 901-906.
(17) Jonikas, M. C., et al. (2009) Comprehensive characterization of genes required for protein folding in the endoplasmic reticulum. Science 323 (5922), 1693-1697.

(18) Howell, G. R., et al. (2007) Mutation of a ubiquitously expressed mouse transmembrane protein (Tapt1) causes specific skeletal homeotic transformations. Genetics 175 (2), 699-707.

(19) Plouffe, D., et al. (2008) In silico activity profiling reveals the mechanism of action of antimalarials discovered in a high-throughput screen. Proc. Natl. Acad. Sci. U.S.A. 105 (26), 9059-9064.

(20) Spangenberg, T., et al. (2013) The open access malaria box: a drug discovery catalyst for neglected diseases. PLoS One 8 (6), e62906.

(21) Bowman, J. D., et al. (2014) Antiapicoplast and gametocytocidal screening to identify the mechanisms of action of compounds within the malaria box. Antimicrob. Agents Chemother. 58 (2), 811-819.

(22) Duffy, S., and Avery, V. M. (2013) Identification of inhibitors of Plasmodium falciparum gametocyte development. Malar. J. 12, 408.

(23) Meister, S., et al. (2011) Imaging of Plasmodium liver stages to drive next-generation antimalarial drug discovery. Science 334 (6061), $1372-1377$.

(24) Plouffe, D. M., et al. (2016) High-throughput assay and discovery of small molecules that interrupt malaria transmission. Cell Host Microbe 19 (1), 114-126.

(25) Ruecker, A., et al. (2014) A male and female gametocyte functional viability assay to identify biologically relevant malaria transmission-blocking drugs. Antimicrob. Agents Chemother. 58 (12), 7292-7302.

(26) Sun, W., et al. (2014) Chemical signatures and new drug targets for gametocytocidal drug development. Sci. Rep. 4, 3743.

(27) Linares, M., et al. (2015) Identifying rapidly parasiticidal antimalarial drugs using a simple and reliable in vitro parasite viability fast assay. Malar. J. 14 (1), 441.

(28) Flannery, E. L., Fidock, D. A., and Winzeler, E. A. (2013) Using genetic methods to define the targets of compounds with antimalarial activity. J. Med. Chem. 56 (20), 7761-7771.

(29) Bozdech, Z., et al. (2003) The transcriptome of the intraerythrocytic developmental cycle of Plasmodium falciparum. PLoS Biol. 1 (1), E5.

(30) Gagaring, K. B. R., Francek, C., Chen, Z., Buenviaje, J., Plouffe, D., Winzeler, E., Brinker, A., Diagana, T., Taylor, J., Glynne, R., Chatterjee, A., Kuhen, K. Genomics Institute of the Novartis Research Foundation (GNF): 10675 John Jay Hopkins Drive, San Diego CA 92121, USA, and Novartis Institute for Tropical Disease, 10 Biopolis Road, Chromos 05-01, 138670 Singapore.

(31) Le Roch, K. G., et al. (2003) Discovery of gene function by expression profiling of the malaria parasite life cycle. Science 301 (5639), 1503-1508.

(32) Llinás, M., et al. (2006) Comparative whole genome transcriptome analysis of three Plasmodium falciparum strains. Nucleic Acids Res. 34 (4), 1166-1173.

(33) Nagle, A., et al. (2012) Imidazolopiperazines: lead optimization of the second-generation antimalarial agents. J. Med. Chem. 55 (9), 4244-4273.

(34) Wu, T. N., et al. (2011) J. Med. Chem. 54, 5116.

(35) Petersen, I., Eastman, R., and Lanzer, M. (2011) Drug-resistant malaria: molecular mechanisms and implications for public health. FEBS Lett. 585 (11), 1551-1562.

(36) Bellanca, S., et al. (2014) Multiple drugs compete for transport via the Plasmodium falciparum chloroquine resistance transporter at distinct but interdependent sites. J. Biol. Chem. 289 (52), 3633636351.

(37) Patel, V., et al. (2008) Identification and characterization of small molecule inhibitors of Plasmodium falciparum dihydroorotate dehydrogenase. J. Biol. Chem. 283 (50), 35078-35085.

(38) Booker, M. L., et al. (2010) Novel inhibitors of Plasmodium falciparum dihydroorotate dehydrogenase with anti-malarial activity in the mouse model. J. Biol. Chem. 285 (43), 33054-33064.

(39) Ross, L. S., et al. (2014) In vitro resistance selections for Plasmodium falciparum dihydroorotate dehydrogenase inhibitors give 
mutants with multiple point mutations in the drug-binding site and altered growth. J. Biol. Chem. 289, 17980.

(40) Gujjar, R., et al. (2009) Identification of a metabolically stable triazolopyrimidine-based dihydroorotate dehydrogenase inhibitor with antimalarial activity in mice. J. Med. Chem. 52 (7), 1864-1872.

(41) Phillips, M. A., et al. (2008) Triazolopyrimidine-based dihydroorotate dehydrogenase inhibitors with potent and selective activity against the malaria parasite Plasmodium falciparum. J. Med. Chem. 51 (12), 3649-3653.

(42) Lukens, A. K. (2014) Proc. Natl. Acad. Sci. U.S.A. 111 (2), 799804.

(43) Spillman, N. J., and Kirk, K. (2015) The malaria parasite cation ATPase PfATP4 and its role in the mechanism of action of a new arsenal of antimalarial drugs. Int. J. Parasitol.: Drugs Drug Resist. 5 (3), $149-162$.

(44) Fry, M., and Pudney, M. (1992) Site of action of the antimalarial hydroxynaphthoquinone, 2-[trans-4-(4'-chlorophenyl)cyclohexyl]-3hydroxy-1,4-naphthoquinone (566C80). Biochem. Pharmacol. 43 (7), $1545-1553$.

(45) Srivastava, I. K., et al. (1999) Resistance mutations reveal the atovaquone-binding domain of cytochrome $\mathrm{b}$ in malaria parasites. Mol. Microbiol. 33 (4), 704-711.

(46) Nam, T. G., et al. (2011) A chemical genomic analysis of decoquinate, a Plasmodium falciparum cytochrome $\mathrm{b}$ inhibitor. ACS Chem. Biol. 6 (11), 1214-1222.

(47) Dong, C., et al. (2011) Identification and validation of tetracyclic benzothiazepines as Plasmodium falciparum cytochrome bc(1) inhibitors. Chem. Biol. 18 (12), 1602-1610.

(48) Bueno, J. M., et al. (2012) Exploration of 4(1H)-pyridones as a novel family of potent antimalarial inhibitors of the plasmodial cytochrome bc1. Future Med. Chem. 4 (18), 2311-2323.

(49) Lukens, A. K., et al. (2015) Diversity-oriented synthesis probe targets Plasmodium falciparum cytochrome $\mathrm{b}$ ubiquinone reduction site and synergizes with oxidation site inhibitors. J. Infect. Dis. 211 (7), 1097-1103.

(50) Nilsen, A., et al. (2013) Quinolone-3-diarylethers: a new class of antimalarial drug. Sci. Transl. Med. 5 (177), 177 ra37.

(51) McNamara, C. W., et al. (2013) Targeting Plasmodium PI(4)K to eliminate malaria. Nature 504 (7479), 248-253.

(52) Ghidelli-Disse, S., et al. (2014) Identification of Plasmodium PI4 kinase as target of MMV390048 by chemoproteomics. Malar. J. 13 (Suppl. 1), P38.

(53) Spitzmuller, A., and Mestres, J. (2013) Prediction of the P. falciparum target space relevant to malaria drug discovery. PLoS Comput. Biol. 9 (10), e1003257.

(54) Aurrecoechea, C. (2009) PlasmoDB: a functional genomic database for malaria parasites. Nucleic Acids Res. 37, D539.

(55) (2015) The Pf3K Project: pilot data release 4; http://www. malariagen.net/data/pf3k-4.

(56) Eastman, R. T., et al. (2011) Piperaquine resistance is associated with a copy number variation on chromosome 5 in drug-pressured Plasmodium falciparum parasites. Antimicrob. Agents Chemother. 55 (8), 3908-3916.

(57) Flannery, E. L., et al. (2015) Mutations in the P-type cationtransporter ATPase 4, PfATP4, mediate resistance to both aminopyrazole and spiroindolone antimalarials. ACS Chem. Biol. 10 (2), 413-420.

(58) Rathod, P. K., McErlean, T., and Lee, P. C. (1997) Variations in frequencies of drug resistance in Plasmodium falciparum. Proc. Natl. Acad. Sci. U.S.A. 94 (17), 9389-9393.

(59) Ding, X., Ubben, D., and Wells, T. (2012) A framework for assessing the risk of resistance for anti-malarials in development. Malar. J. 11 (1), 292.

(60) Chiodini, P. L., et al. (1995) Evaluation of atovaquone in the treatment of patients with uncomplicated Plasmodium falciparum malaria. J. Antimicrob. Chemother. 36 (6), 1073-1078.

(61) Looareesuwan, S., et al. (1996) Clinical studies of atovaquone, alone or in combination with other antimalarial drugs, for treatment of acute uncomplicated malaria in Thailand. Am. J. Trop. Med. Hyg. 54 (1), 62-66.

(62) Korsinczky, M., et al. (2000) Mutations in Plasmodium falciparum cytochrome $\mathrm{b}$ that are associated with atovaquone resistance are located at a putative drug-binding site. Antimicrob. Agents Chemother. 44 (8), 2100-2108.

(63) McKeage, K., and Scott, L. (2003) Atovaquone/proguanil: a review of its use for the prophylaxis of Plasmodium falciparum malaria. Drugs 63 (6), 597-623.

(64) Babbitt, S. E., et al. (2012) Plasmodium falciparum responds to amino acid starvation by entering into a hibernatory state. Proc. Natl. Acad. Sci. U.S.A. 109 (47), E3278-E3287.

(65) Trager, W., and Jensen, J. B. (1976) Science 193 (4254), 673.

(66) Silvie, O., et al. (2002) Hepatocyte CD81 is required for Plasmodium falciparum and Plasmodium yoelii sporozoite infectivity. Nat. Med. 9 (1), 93-96.

(67) Manary, M. J., et al. (2014) Identification of pathogen genomic variants through an integrated pipeline. BMC Bioinf. 15 (1), 1-14.

(68) Johnson, J. D., et al. (2007) Assessment and continued validation of the malaria SYBR Green I-based fluorescence assay for use in malaria drug screening. Antimicrob. Agents Chemother. 51 (6), 1926-1933.

(69) Witkowski, B., et al. (2013) Novel phenotypic assays for the detection of artemisinin-resistant Plasmodium falciparum malaria in Cambodia: in-vitro and ex-vivo drug-response studies. Lancet Infect. Dis. 13 (12), 1043-1049. 